\title{
Psychiatric liaison in palliative care
}

\author{
Simon Dein
}

Abstract Palliative care is the active, total care of patients whose disease is not responsive to curative treatment. Psychological problems such as depressive illness, anxiety, delirium, problems coping and body image disturbances are common in the palliative care setting, although they are often missed. A full assessment of these patients should take into account physical, emotional and spiritual factors; therapeutic work should include the patient's family. Pharmacological (antidepressants, anxiolytics and antipsychotics) and cognitive-behavioural treatments are often effective in allaying distress in this group of patients, and can improve coping skills and quality of life. Liaison psychiatrists have a role in teaching palliative care staff to recognise psychiatric disorders. A number of case studies are presented to illustrate these points.

The World Health Organization (1990) defines palliative care as:

'The active total care of patients whose disease is not
responsive to curative treatment. Control of pain, of
other symptoms, and of psychological, social and
spiritual problems is paramount. The goal of palliative
care is achievement of the best quality of life for
patients and their families. Many aspects of palliative
care are also applicable earlier in the course of the
illness in conjunction with anticancer treatment.'

Although the term 'terminal care' is often used in relation to this group of patients, it is not synonymous with 'palliative care' and not all patients face imminent death. Consultation-liaison psychiatry in this area can be very challenging, as it involves working with patients who have a poor prognosis and their families. The management of patients in palliative care often poses ethical dilemmas that may differ significantly from those posed by work in general psychiatry. For example, to what extent should patients be actively treated? When should treatment stop? Is the patient competent to make these decisions? If not, who can make them? Is withholding treatment different from actively killing the patient? Liaison psychiatry also provides the opportunity to work with specialist multi-disciplinary teams that deal with physical, emotional and spiritual aspects of illness. Psychiatric advice may be sought in relation to a number of problems, including communicating bad news, dealing with psychiatric disorders, coping with illness, managing treatment-resistant physical symptoms, issues of distorted body image, and teaching staff to recognise and treat psychiatric illness.

\section{Background}

The modern palliative care movement originated with the establishment of St Christopher's Hospice in London in 1967 by Dame Cecily Saunders. Although most terminally ill patients wish to die at home, only $25 \%$ do so. Over $90 \%$ of cancer patients spend some time in hospital during their final year; $55 \%$ die in hospital and $17 \%$ die in a hospice (O'Neill \& Rodway, 1999). Most hospices now provide home care services, and many independent home care teams liaise closely with primary care. In fact, most palliative care is provided by general practitioners, with palliative care consultants dealing with in-patients or difficult symptoms. Unlike the rest of the health service, most hospices are funded by the voluntary sector, with only partial financial support from the National Health Service. Palliative care teams traditionally manage patients with advanced cancer. However, some also manage AIDS and advanced neurological diseases (such as motor neuron disease, multiple sclerosis and Huntington's chorea). Recently, patients with advanced cardiac disease (particularly congestive cardiac failure) and advanced respiratory disease (chronic obstructive airway disease) have been referred to palliative care teams. In this paper, I focus on the palliative care of cancer patients, which is my area of expertise.

Simon Dein is a senior lecturer at University College London and a consultant psychiatrist at Princess Alexandra Hospital (The Derwent Centre, Hamstel Road, Harlow, Essex CM20 1QX, UK. Fax: 01279 454018; e-mail: jenny.lloyd@nemhpt.nhs.uk). He has an interest in the relation between psychiatry and palliative care and holds a diploma in palliative medicine from Cardiff University. 
Physical symptoms are extremely prevalent in patients with advanced cancer. They include pain, fatigue, somnolence or mental clouding and gastrointestinal problems (Box 1), with most patients experiencing multiple physical and psychological symptoms (Portenoy et al, 1994; Chochinov \& Breitbart, 2000). On account of their advanced medical illnesses, patients are often on several different types of medication. The potential for adverse reactions to psychotropic drugs should always be borne in mind, along with the fact that compromised renal and hepatic function might alter the pharmacodynamics of certain drugs. A full assessment of these patients involves taking into account physical, psychological and existential factors, all of which can mutually influence each other.

\section{Psychological and spiritual adjustment to life-threatening illness}

By the time patients reach the palliative care stage, they have typically gone through a process of investigation, diagnosis and treatment, with varying degrees of pain and trauma, dependency and disfigurement. Following diagnosis of life-threatening illness, many patients experience shock punctuated by periods of dysphoria and anxiety, fatalism and grief (Massie, 1989). Adjustment disorders are the most common psychiatric diagnosis (White \& Macleod, 2002). Only a minority of cancer patients develop psychiatric illness. However, psychological and social problems, including difficulties with employment and finance, are common.

Kubler-Ross (1970) argues that there are five stages to patients' reaction to a terminal diagnosis: denial, anger, bargaining, depression and acceptance. It is now recognised that these emotions can occur simultaneously, and are not exhibited by all patients. Fears that typically accompany a diagnosis of terminal disease include loss of bodily functions, disfiguring treatments, loss of autonomy and independence, abandonment and isolation, becoming a burden, financial strain, the end of known existence and uncontrollable pain.

Patients with life-threatening illness often experience a number of existential concerns relating to questions such as 'Why me?' or 'What have I done to deserve this?' They might have difficulty finding meaning in their suffering. The illness might cause them to question their relationship with God (how could God allow this to happen?), even to the extent of losing their faith. There might be issues about death and dying, and patients often seek reassurance that there is an afterlife. Spiritual issues
Box 1 Common symptoms in palliative care patients

- Pain

- Fatigue

- Somnolence

- Gastrointestinal problems

about meaning can have a significant effect on mood (Austin \& Jennings, 1993) and patients might express these spiritual concerns to doctors, who frequently feel unable to answer these questions and refer them to clergymen. However, a full spiritual assessment of the patient is important. This should ask what the illness means to the patient and whether there are any issues relating to God or dying. At times, it can be difficult to distinguish spiritual distress from agitated depression.

\section{Denial as a response to illness}

Patients vary in their ways of coping, which can range from frank denial to stoical acceptance. Five common adjustment styles in relation to the diagnosis of a life-threatening illness have been described that influence the emotional reaction to illness (Moorey \& Greer, 1989). These include a fighting spirit, denial, fatalism, helplessness / hopelessness and anxious preoccupation (where the patient is constantly worrying about the illness and, in the case of cancer, the possibility of recurrence). Psychiatric intervention might be required if adjustment reactions become extreme and significantly impair the quality of life. A particularly difficult problem is that of denial. Patients often use denial as a defence when the truth is too painful to bear, and it should not be challenged unless it creates serious problems for the patient or relatives. Some patients die in a state of denial. When challenging denial, it is important to be gentle, so that fragile defences are not disrupted but it is equally important to be firm enough that any awareness can be explored and developed. Denial can be a fluctuating process and can vary throughout the course of the illness - one moment, the patient accepts the illness only to deny it at a later time. Physicians should begin by asking patients what has happened since their illness was first discovered and how they felt at each key point, for instance, when they first developed symptoms, saw a specialist, were tested and were informed about the results. It is important to explore perceptions of what is wrong.

\footnotetext{
Case study 1

A 38-year-old man with gastric cancer was referred for psychiatric assessment when the team became
} 
concerned that he denied that he had cancer and was dying. He agreed that he had an ulcer, but thought it would disappear. Following several discussions with him, he agreed that his gastrointestinal symptoms were not improving and that his ulcer was gradually getting worse. Until the time of his death, 5 weeks later, he never used the word 'cancer', but admitted that there was a serious problem and agreed to take symptomatic treatment (although not palliative chemotherapy). The diagnosis of cancer was never forced upon him.

\section{Psychiatric disorders in palliative care}

The prevalence of psychiatric disorders in terminally ill patients is high, and patients with advanced disease and untreated pain are particularly vulnerable (Glover et al, 1995; Williamson \& Schultz, 1995). Depressive disorders might be difficult to diagnose in those with advanced disease, and are commonly missed (Box 2). Studies on the prevalence of depressive disorders in advanced illness have deployed clinician assessments, single-item screening questionnaires, screening questionnaires (such as the Hospital Anxiety and Depression Scale and the Beck Depression Inventory) and diagnostic interviews, but are flawed on account of small numbers of patients and very high non-participation rates (Hotopf, 2002). The cut-off point between a normal reaction, adjustment disorder and depressive disorder varies between studies. The prevalence of major depression in those with advanced cancer is estimated to be between $5-15 \%$, even when the most stringent criteria are used. Another $10-15 \%$ present with symptoms that are less severe. Somatic symptoms that are characteristically used to diagnose depressive disorders, such as anorexia and anergia, might be due to physical illness and are not useful when diagnosing depression in this population. Most authors would consider anhedonia, which is pervasive and extends to a loss of interest or pleasure in almost all activities, to be a valid criterion for the diagnosis of depression (Lynch, 1995; Passik \& Breitbart, 1996).

There is an increased risk of suicide in cancer patients, especially in those with advanced disease, a history of depression, hopelessness, loss of control,

\section{Box 2 Why depression is missed}

- Assumption that symptoms are a natural reaction to illness

- Attributing physical symptoms to illness

- Patient's unwillingness to report symptoms

- Doctor's difficulty in asking about symptoms uncontrolled pain and fatigue. Suicidal talk in those receiving palliative care is by no means inevitable, and not all those who discuss suicide wish to die. There are patients who talk of the option of suicide as one way of terminating their suffering and regaining control, but who do not consider killing themselves as a serious option. This must be taken into account in the risk assessment.

The wish to die in terminally ill patients may fluctuate over time and may be correlated with levels of depression and anxiety, pain and dypsnoea (Chochinov et al, 1995; Chochinov et al, 1999). Any legislation in relation to euthanasia must take account of this. Treating these symptoms with appropriate palliative care might significantly reduce the desire for death. A particularly difficult decision relates to the use of the Mental Health Act 1983 among suicidal patients with a significantly limited life expectancy. The question of rational suicide often arises in the assessment. Can patients with terminal illnesses rationally conclude that life is not worth living, and should they be allowed to kill themselves? The decision to compulsorily treat a patient might be very difficult, and must take into account the views of the family.

\footnotetext{
Case study 2

A 25-year-old man with advanced AIDS (pneumocystic pneumonia and Kaposi's sarcoma) was admitted to a medical ward following a large overdose of paracetamol and alcohol. On assessment, he was hopeless and expressed the desire to die to 'put himself out of his misery'. He admitted to having felt low for some time and was weak, lacking in energy and considerably underweight. He was fully aware of his diagnosis and prognosis (very poor). He asked that we allow him to go home to die. Was this man clinically depressed? Was this an appropriate response to his illness? A mental health assessment was carried out and, after discussion with his family, he was discharged home, although we recognised that he was at serious risk of suicide. The difficulty of placing him on a mental health section was raised. He died 3 weeks later at home from pneumonia.
}

\section{The management of depression}

Before beginning treatment with drugs, renal and hepatic functioning should be assessed, as both might be significantly impaired in those with advanced cancer. It is also essential to take into account other medications that the patient is taking, since there might be significant interaction between these and psychotropic medication. There is wellestablished evidence that antidepressants are effective in treating depression in cancer patients (Massie, 1990), especially where it is difficult to differentiate between depression and adjustment 
disorder. The perceived prognosis and the timeframe of antidepressant agents must be taken into account before starting an antidepressant. The patient who has only weeks to live might benefit from a rapid-acting psychostimulant (Dein \& George, 2002) such as dexamphetamine or methylphenidate. Tricyclic antidepressants have a sideeffect profile that can be troublesome for palliative care patients and exacerbate existing symptoms. These include constipation, dry mouth and urinary retention, and may precipitate anticholinergic delirium. Amitryptyline can be useful where there is associated neuropathic pain and in those who are agitated. Among those with advanced disease, tricyclics should be started at low doses.

Similarly, selective serotonin reuptake inhibitors are useful antidepressants, although there are no specific studies of their use in terminally ill patients. For these patients, therapy should be initiated at half the usual starting dose. Fluoxetine is well tolerated by severely medically ill patients, although it can cause sweating, nausea and insomnia. A recent double-blind trial using fluoxetine (Razavi et al, 1996) in patients with cancer did not report any significant change in baseline scores on the Hospital Anxiety and Depression Scale. It is available as an elixir, and therefore easier to administer in the severely medically ill, although there is no evidence to suggest that it is better than any other antidepressant. I have found lofepramine to be a relatively effective and well-tolerated antidepressant.

\section{Case study 3 \\ A 44-year-old man with advanced bladder cancer and pelvic spread took to his bed complaining of extreme weakness and tiredness. Although taking reasonable doses of opiates, the physicians treating him felt that his tiredness and weakness could not be fully accounted for by an organic condition and requested a psychiatric consultation. The patient admitted to feeling low, but rationalised it by saying 'wouldn't you feel low in my position?'. He admitted to loss of interest and his sleep was disturbed, partly because of persistent pain in his perineum thought to be neuropathic in nature. On balance, it was thought that he was probably clinically depressed, in view of his pervasive low mood and anhedonia. He was started on a small dose of amitryptyline $(25 \mathrm{mg})$ and this was slowly increased to $75 \mathrm{mg}$ at night. Amitryptyline was chosen, as it has been shown to be effective in neuropathic pain. After a couple of weeks, he showed a mild improvement in his mood and was able to spend time out of bed. His pain was also reduced in intensity.}

\section{Anxiety}

The evaluation of anxiety is a common reason for psychiatric referral. Anxiety levels change over the

\section{Box 3 Causes of anxiety in palliative care}

\begin{tabular}{|c|c|}
\hline Psychiatric & Organic \\
\hline Agitation & Hypoxia \\
\hline Depression & Sepsis \\
\hline $\begin{array}{l}\text { Post-traumatic } \\
\text { stress disorder }\end{array}$ & Pain \\
\hline Existential & Iatrogenic \\
\hline $\begin{array}{l}\text { General concerns about } \\
\text { dependency, disfigurement, } \\
\text { life, death and existence }\end{array}$ & Drug side-effects \\
\hline
\end{tabular}

course of an illness, and might increase with the diagnosis of cancer, peak prior to surgical interventions and frequently remain high, only slowly declining during the post-operative years (Maguire et al, 1978). Increased anxiety might also occur prior to palliative chemotherapy (Peck \& Bowlan, 1977; Kvale, 1993). It is often part of another psychiatric syndrome such as depressive disorder, posttraumatic stress disorder (Alter et al, 1996) or delirium. The somatic manifestations of anxiety often overshadow the psychological or cognitive manifestations and may be difficult to differentiate from physical symptoms of the disease such as tachycardia and shortness of breath. The anxiety itself might arise from an organic complication of the illness such as hypoxia, sepsis, poorly controlled pain, dehydration or drug withdrawal (e.g. opiates) or it might be a drug side-effect such as akathisia (Box 3). All patients need a full medical assessment. Existential issues relating to death and dying, dependency and disfigurement also commonly play a role in anxiety and might need to be addressed, possibly in collaboration with religious advisers.

In terms of drug treatment for anxiety, the shorteracting benzodiazepines such as lorazepam and oxazepam (orally or parenterally) are safest, as the patients often have reduced hepatic metabolism. All benzodiazepines can cause somnolence and confusion, especially in the elderly and those with advanced disease. Acute severe anxiety may present as severe agitation or panic attacks and require emergency treatment. It can be treated with oral or sublingual lorazepam, or with intravenous or subcutaneous midazolam. Cognitive-behavioural therapies, including relaxation and distraction, have a role to play in anxiety management.

\section{Neuropsychiatric disorders}

Delirium is common in palliative care settings. Pereira et al (1997) found the prevalence of cognitive impairment to be $44 \%$ in cancer in-patients, rising 
to $62 \%$ shortly before death. Two subtypes are seen in the palliative care setting, which are differentiated by the level of arousal and psychomotor activity: the hyperactive and the hypoactive (Lipowski, 1983). The former is characterised by hallucinations, delusions, agitation and disorientation, whereas the latter is characterised by confusion and sedation. The clinician encountering a delirious patient should always formulate a list of differential diagnoses as to the likely aetiology, although there is some debate regarding the extent of diagnostic evaluation that should be carried out in this population group.

Diagnostic evaluation is limited by practical constraints such as the setting (home, hospice), patient comfort and the doctor's perception of prognosis. The aetiology is usually multi-factorial and a specific aetiology is discovered in less than $50 \%$ of terminally ill patients with delirium (Breura et al, 1992). During the terminal phase, the delirium might be an irreversible, integral part of the dying process. Hallucinations and delusions involving dead relatives may in fact be pleasant and comforting for the patient.

Following a full physical examination for dehydration, sepsis and major organic failure, laboratory tests for metabolic abnormalities such as hypercalcaemia should be carried out. All medications possibly contributing to the delirium (especially opiates) should be reviewed. In the relatively fit patient, it might be deemed necessary to perform computed tomography or magnetic resonance imaging scans to detect possible metastases from the lung, breast or alimentary tract. These metastases may require specific treatments in themselves. Some cancers (e.g. of the lungs or ovaries) produce paraneoplastic syndromes, including confusion. The management of delirium should be symptomatic, and supportive measures are important. Maintaining a normal fluid and electrolyte balance, providing adequate nutrition and reducing anxiety and disorientation are all important. A well-lit room with familiar objects, a visible clock or a calendar can also be helpful.

A number of neuroleptics are effective in the management of psychotic symptoms in patients with advanced cancer and they are commonly used to treat delirium. Among the standard antipsychotic drugs, haloperidol is the drug of choice in medically ill patients and those with advanced disease. In low doses of 1-3 mg/day, it is effective in targeting agitation, paranoia and hallucinations (Akechi et al, 1996) and it has the advantage of being an effective treatment for nausea and vomiting, which are also common in the palliative care setting. More recently, risperidone ( $0.5-1 \mathrm{mg}$ twice daily) has been used to treat severe delirium in those who are intolerant to the extrapyramidal side-effects of classic neuroleptics. Methotrimeprazine is especially useful on account of its associated analgesic properties. Both haloperidol and methotrimeprazine can be given in a syringe driver to patients who can no longer take oral medication.

\section{Case study 4 \\ Psychiatric advice was sought on a 60-year-old woman who, 3 months previously, was diagnosed with stage III breast cancer. Over 2 days, she had become confused and agitated and was wandering about aimlessly. On mental state examination, she was distressed, agitated and disoriented for time and place and appeared to be experiencing visual hallucinations. Although her neurological examin- ation was unremarkable, a computed tomography scan revealed multiple metastases in her frontal and temporal lobes. She was commenced on dexa- methasone (to reduce cerebral pressure), but became more confused and agitated. Her symptoms were managed with methotrimeprazine in a syringe driver and regular oral lorazepam. She settled quickly, and died peacefully a week later in hospital.}

\section{Coping with illness}

It is the implications of the illness for the patient that are important. The emotional response is determined to some extent by the patient's view of the diagnosis, perceived control over the illness and the prognosis. It is often issues of unpredictability and uncertainty that dominate in the patient's mind (Moorey \& Greer, 1989). It is not only the threat to survival that concerns the patient; there is often a threat to self-image ('Will people find me attractive without a breast?') and a disruption of social roles, including work. For those whose self-image is dependent on working, the loss of this role can be devastating.

Psychological techniques have been developed and used in conjunction with more traditional medical interventions to alleviate symptoms and the disturbed thoughts, mood and behavior commonly associated with dying. Many patients benefit from discussion and problem-solving techniques, education and reassurance about the common threats and losses associated with illness, and 'empathic listening to [their] views, uncertainties and beliefs about the illness' (Peveler et al, 2002). Cognitive-behavioural therapy is carried out over a number of sessions, each lasting up to an hour. The patient's spouse may be involved in the sessions and a large part of the therapy is done at home as 'homework'. At the beginning of therapy, the rationale of the technique is explained to the patient. The patient should be informed that their response to the cancer determines how they feel and cope. 
The therapist starts by identifying problems and the way that the patient deals with them. Negative automatic thoughts are identified, which themselves are unhelpful and lead to distress. Negative automatic thoughts are not necessarily false and may be accurate in some cases. They become dysfunctional when the patient cannot stop ruminating on them and they interfere excessively with the person's life. Common cognitions include 'I know I am going to die from cancer' (which may be accurate), 'There is no point in doing anything', 'I'm so unattractive after losing my breast'. The therapist and the patient together examine in some detail the rationality of the thoughts and test them against reality, constantly looking for alternatives. The first step is to learn to recognise these thoughts. Patients are encouraged to monitor and record their thoughts and examine how realistic they are. If they are unrealistic, they can be challenged. The therapy also attempts to improve the quality of life and regain a sense of mastery by planning activities that the patient enjoys.

\section{Case study 5 \\ A 30-year-old woman with metastatic breast cancer (although with only one bony secondary) was referred by her oncologist because she was agitated and preoccupied with her cancer spreading. She frequently checked herself, and phoned her surgeon almost daily to seek reassurance. Much of her daily routine involved examining herself for signs of recurrence. She was hopeless, repetitively saying 'What's the point of doing anything if I am going to die?' Using distraction techniques (listening to pleasant music), relaxation, mastery (getting her to garden, an activity she previously enjoyed) and reattribution (learning not to attribute every symptom to her cancer) she slowly became less agitated and was able to continue with her life.}

\section{Psychological management of physical symptoms}

The psychiatrist might be asked about the management of treatment-resistant physical symptoms such as pain and vomiting, or physical symptoms believed to have a significant psychological overlay. Cognitive therapy can help patients to reinterpret noxious sensations as less intense, to relabel physical symptoms in other terms and to engage in coping mechanisms (Meyer \& Mark, 1995). It is well recognised that psychological factors such as anxiety, expectancy, cognitive appraisal, selfefficacy and perceived control can influence the reporting and experience of physical symptoms such as nausea and pain (Hill et al, 1986). Techniques such as relaxation, distraction, attentional training, guided imagery, controlled breathing and hypnosis might significantly influence the experience of pain. Another useful technique is cognitive restructuring, whereby the symptom is reconceptualised. The patient is encouraged to identify and change stress-inducing thoughts and feelings associated with symptoms. For instance, the statement 'I feel as if I can't take the pain any more' is modified to the statement 'I have coped with this symptom before and I can do it again'.

\section{Body-image disturbances}

Advanced physical illness has a significant effect on body image, either from the disease itself (e.g. massive ascites) or, more commonly, from treatments such as mastectomy, colostomy or chemotherapy (a side-effect of which is alopecia). The distorted body image can cause significant psychological distress and interfere with everyday activities and relationships. Problems might relate to perceived attractiveness as a person and as a sexual partner, and it is not surprising that sexual problems are common in this population (Gill, 1997). Those with body-image disturbances associated with avoidance of looking at the results of surgery (for example, of mastectomy) are at particularly high risk of mood disorder (Maguire et al, 1983). Similarly, up to $32 \%$ of those with a stoma following large bowel surgery become clinically depressed (Thomas et al, 1987). Body image disturbances are a common reason for psychiatric referral and often respond well to cognitive approaches.

\section{Case study 6}

A 50-year-old woman with advanced breast cancer was referred for psychiatric evaluation. Since her mastectomy 2 years earlier, she had been unable to undress in front of her husband, with consequent detrimental effects on their relationship. She could not bear her husband to see her scar, and felt unfeminine and mutilated. Her husband stated that even without her breast he still found her attractive, but she was distancing him. During several sessions of desensitisation, she was asked to imagine how her husband would respond when he saw her naked. Slowly, she was able to relax during these fantasies. After several weeks, she allowed her husband to see her in the bath. Shortly afterwards, she started to undress in front of him and there was a significant improvement in their sexual relationship.

\section{The family as the unit of care}

The dying process usually has a significant effect on the family, who are considered to be the unit of care by hospice staff. They can powerfully affect the 
patient's mood, and are themselves at risk of depression and anxiety. Issues of unresolved anger and dependency may appear at this time that can exacerbate psychological distress and influence the subsequent course of bereavement. A psychiatric opinion may be sought in relation to the management of these conflicts, particularly in those thought to be at significant risk of abnormal grief reactions (Dein, 2000). There is scope for doing family work with dying patients. A common problem is that of collusion, whereby the family does not want the patient to be told the truth about the diagnosis. This can be a difficult problem, and it must be handled with great sensitivity. Generally, the attitude of those working in palliative care is that the patient has a right to know if he or she asks. At other times, the family might refuse to allow active treatment to be stopped, despite the wishes of the patient.

\section{Case study 7}

Advice was sought about a family that the hospice staff saw as 'difficult'. Although the patient was terminally ill, confused and not drinking or eating, the family demanded that he was given a drip, against the advice of hospice staff (it is generally the policy of hospices not to drip-feed patients in the last few days of life). During several meetings with the family, it emerged that they had great problems letting go of him and overcoming their significant dependency on him. These issues were discussed in detail, and he died several days later without being drip-fed. His daughter admitted that these discussions had helped her to better cope with the grief after his death.

\section{Education of palliative care staff about psychiatric disorders}

The low pick-up rate of psychiatric disorders in medically ill patients has been frequently reported (Feldman et al, 1987). The liaison psychiatrist plays an important role in teaching staff about the symptoms and treatments of various psychiatric disorders. In particular, he or she can demolish the 'myth' that emotional symptoms are inevitable reactions to the illness and that they cannot be treated. Staff can be taught to recognise these symptoms and manage them. Although staff burnout in palliative care is less common than in other medical specialities, at times staff do require support, particularly in relation to issues of loss, death and dying. The liaison psychiatrist can also discuss with staff their responses to patients and relatives, supporting them and pointing out signs of burnout and stress-related reactions (Graham et al, 1996).

\section{Conclusions}

The psychiatrist can provide expertise in a number of areas of palliative care. To date, the number of palliative care services with psychiatric liaison is unknown. However, the statement is commonly made by palliative care staff that a psychiatrist would be a valuable addition to the palliative care team. Those working in this area must learn to recognise both expressions of psychiatric disorder in patients with severe physical illness and normal adjustments to dying. There is no doubt that palliative care can be highly satisfying for the psychiatrist and significantly improve the quality of life for patients. However, the work can be emotionally challenging, especially coping with the fact that patients with whom you have built up a good relationship die in your care.

\section{References}

Akechi, T., Uchitomi, Y., Okamura, H., et al (1996) Usage of haloperidol for delirium in cancer patients. Supportive Care Cancer, 4, 390-392.

Alter, C. L., Pelcovitz, D., Axelrod, A., et al (1996) The identification of PTSD in cancer survivors. Psychosomatics, $37,137-143$.

Austin, D. \& Jennings, C. J. (1993) Grief and religious belief: does belief moderate depression? Death Studies, 17, 487496.

Breura, E., Miller, L., Mattkellan, J., et al (1992) Cognitive failure in patients with terminal cancer: prospective study. Journal of Pain and Symptom Management, 7, 192-195.

Chochinov, H. \& Breitbart, W. (2000) Handbook of Psychiatry in Palliative Medicine. New York: Oxford University Press.

-, Wilson, K. G., Enns, M., et al (1995) Desire for death in the terminally ill. American Journal of Psychiatry, 152, 11851191.

—, Tataryn, D. \& Clinch, J. (1999) Will to live in the terminally ill. Lancet, 354, 816-819.

Dein, S. (2000) The management of psychological distress in terminal illness. Primary Care Psychiatry, 6, 123-126.

- \& George, R. (2002) A place for psychostimulants in palliative care. Journal of Palliative Care, 18, 196-199.

Feldman, E., Mayou, R., Hawton, K., et al (1987) Psychiatric disorder in medical in-patients. Quarterly Journal of Medicine, 63, 405-412.

Gill, P. (1997) Male sexual problems in palliative care. Palliative Care Today, 5, 46-47.

Glover, J., Dibble, S., Dodd, M., et al (1995) Mood states of oncology outpatients: does pain make a difference? Journal of Pain and Symptom Management, 10, 120-128.

Graham, J., Ramirez, A., Cull, A., et al (1996) Job stress and satisfaction among palliative physicians. Palliative Medicine, 10, 185-194.

Hill, H., Saeger, L. C., Chapman, C. R. (1986) Patient controlled analgesia after bone marrow transplantation for cancer. Postgraduate Medicine, August, 33-40.

Hotopf, M. (2002) Depression in advanced disease: a systematic review. Part 1: Prevalence and case finding. Palliative Medicine, 16, 81-97.

Kubler-Ross, E. (1970) On Death and Dying. London: Tavistock Publications.

Kvale, G., Glynmellious, B., Hoffman, K., et al (1993) Prechemotherapy nervousness as a mark of anticipatory nausea: a case of a non-cause predictor. Psycho-Oncology, 2, 33-41. 
Lipowski, J. (1983) Transient cognitive disorders, delirium, acute confusional states in the elderly. American Journal of Psychiatry, 140, 1426-1436.

Lynch, M. E. (1995) The assessment and prevalence of affective disorders in advanced cancer. Journal of Palliative Care, 11, 10-18.

Maguire, G. P., Lee, E., Bevington, D., et al (1978) Psychiatric problems in the first year after mastectomy. BMJ, 1, 963965.

Maguire, P., Brooke, M., Tait, A., et al (1983) Effect of counselling on the psychiatric morbidity associated with mastectomy. BMJ, 281, 1454-1456.

Massie, M. J. (1989) Depression. In Handbook of PsychoOncology: Psychological Care of the Patient with Cancer (eds J. C. Holland \& J. H. Roland), pp. 283-290. New York: Oxford University Press.

Meyer, T. J. \& Mark, M. M. (1995) Effects of psychosocial interventions with adult cancer patients: an analysis of randomised experiments. Health Psychology, 14, 101-108.

Moorey, S. \& Greer, S. (1989) Psychological Therapy for Patients with Cancer: A New Approach. Oxford: Heinemann.

O'Neill, B. \& Rodway, A. (1999) Care in the community. In $A B C$ of Palliative Care (eds M. Fallon \& B. O'Neill), pp. 5862. London: BMJ Books.

Passik, S. D. \& Breitbart, W. S. (1996) Depression in patients with pancreatic carcinoma: diagnostic and treatment issues. Cancer, 78, 615-626.

Peck, A. \& Bowlan, J. (1977) Emotional reactions to radiation treatment. Cancer, 40, 180-184.

Pereira, J., Hanson, J. \& Bruera, E. (1997) The frequency in clinical course of cognitive impairment in patients with terminal cancer. Cancer, 79, 835-841.

Peveler, R., Carson, A. \& Rodin, G. (2002) Depression in medical patients. ABC of psychological medicine. BMJ, 325, 149-152.

Portenoy, R. K., Thaler, H. T., Komblith, A. B., et al (1994) Symptom prevalence characteristics and distress in a cancer population. Quality of Life Research, 3, 183-189.

Razavi, D., Allilaire, J. F., \& Smith, M. (1996) The effect of fluoxetine on anxiety and depression symptoms in cancer patients. Acta Psychiatrica Scandinavica, 94, 205-210.

Thomas, C., Medden, F. \& Jehu, D. (1987) Psychological effects of stomas. II: Factors influencing outcome. Journal of Psychosomatic Research, 31, 317-323.

White, C. \& Macleod, U. (2002) Cancer. ABC of psychological medicine. BMJ, 325, 377-380.

Williamson, G. \& Schultz, R. (1995) Activity restriction mediates the association between pain and depressed affect: a study of younger and older cancer patients. Psychology and Aging, 10, 369-378.

World Health Organization (1990) Cancer Pain Relief and Palliative Care (Technical report, series 804). Geneva: WHO.

\section{Multiple choice questions}

\section{Denial:}

a must always be confronted

b can be reduced with antidepressants

c may be a fluctuating process

$\mathrm{d}$ in some patients remains until death

e should be confronted only if it causes significant problems for the patient or family.
2 Depressive disorders in terminal illness:

a are common in palliative care settings

b are generally picked up

c may be diagnosed from somatic symptoms

d do not respond to antidepressants

e may respond to amphetamines.

3 Delirium in palliative care:

a occurs in over $50 \%$ of patients at some stage in their illness

b may present with sedation

c always responds to neuroleptic medication

d may be irreversible

e may be helped by haloperidol.

4 As regards drug therapy in palliative care:

a lorazepam is useful for acute anxiety

b amitryptyline is useful for neuropathic pain

c risperidone may be useful for psychotic symptoms in terminally ill patients

d methotrimeprazine reduces agitation

e selective serotonin reuptake inhibitors are more effective than tricyclics in the management of depression in terminal illness.

\section{Cognitive-behavioural therapy}

a can help patients with body image disturbance

$b$ is not useful for pain

c cannot be used at the same time as medication

d can improve hopelessness in terminally ill patients

e can help to reduce symptoms of depression in terminal illness. 